\title{
REDUCTION OF MULTIPLE SCATTERING TO SECOND ORDER SCATTERING FOR DETERMINATION OF LIQUID-CLOUD MICROPHYSICAL PROPERTIES
}

\author{
Xiaoying $\mathrm{Cao}^{1}$, Gilles Roy ${ }^{2 *}$ and Grégoire Tremblay ${ }^{3}$ \\ ${ }^{1}$ Lidar Consultant, Ottawa, Canada; ${ }^{2 *}$ RDDC Valcartier 2459 de la Bravoure, Québec, Qc, Canada, \\ gilles.roy@drdc-rddc.gc.ca \\ ${ }^{3}$ AEREX Avionic, Levis, Canada;
}

\begin{abstract}
Under single scattering conditions, water droplets clouds do not depolarize the backscattered light. However, backscattered light from multiple scattering will be depolarized. The level of depolarization is a function of the droplets size and of the cloud extinction coefficient value and its profile; it has also a strong dependency on the lidar field-of-view (FOV) and azimuth angle [1][3]. It has been demonstrated in [4] that the multiple scattering cross polarization signal can be reduced to a second order scattering one using the azimuthal dependency of the cross polarization. In this paper we present how the multiple scattering cross polarization can be reduced to a second order scattering and how this signal is related to the droplet particle size. Monte Carlo simulations for clouds at different ranges and effective radius sizes are performed and compared with the results obtained with an analytical model.
\end{abstract}

\section{AZIMUTHAL CROSS POLARIZATION PATTERN}

Backscattered light from spherical particles does not change the polarization orientation state of a linearly polarized incident light. This particularity allows the discrimination of spherical particles from non-spherical particles. However, under multiple scattering conditions, even spherical particles do generate depolarized signals. In fact, the multiple scattering cross polarization shows an azimuthal pattern. This pattern is easily observed using a gated ICCD camera and a polarized beam splitter as shown in Fig. 1. A linearly polarized parallel laser beam is used. At the reception, after collimation, the cross polarization is imaged on the detector. An adjustable delay is used to image the backscattered light from the cloud from a specific cloud depth. The gate width of the ICCD is typically 10 to $20 \mathrm{~ns}$. Please refer to [1] for relevant details.

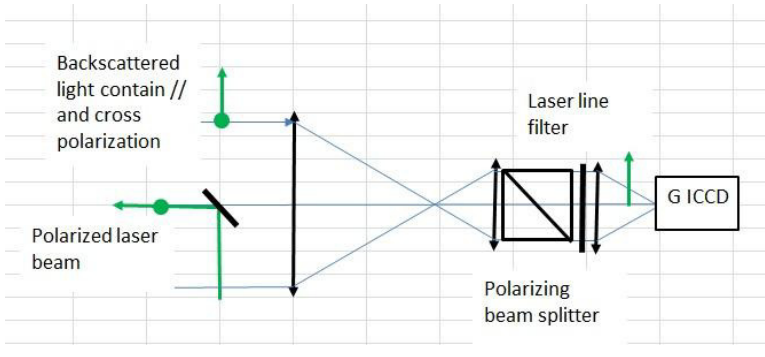

Figure 1. Lidar set up to capture the azimuthal cross polarization. The linearly polarized source is emitted on axis (lower left). The received signal is collimated through a polarizer and the cross polarization is imaged on the camera (right).

In order to better understand the mechanism of polarized multiple scattering, Monte Carlo (MC) simulation has been performed. The Undique MC simulator is a multithreaded software based on the Bohren and Huffman Mie scattering algorithm. The simulator reproduces the characteristics of a Flash Lidar system. It consists of an emitter/receiver system, a target and a propagation range including aerosols of various properties. The particularity of the Undique MC is its capability to image the scattered light on a detector array [5]. Fig. 2 shows the azimuthal pattern obtained for the simulation of a penetration of 50 $\mathrm{m}$ in a cloud of constant extinction $0.03 \mathrm{~m}^{-1}$ starting at $1000 \mathrm{~m}$. The image at the top contains all the scattering orders, while the image at the bottom contains second order scattering only. The second order scattering cross polarization follows a $\operatorname{Cos}(4 \varphi)$ pattern; it means there is no secondorder scattered energy for azimuths equal to $0^{\circ}$, $90^{\circ}, 180^{\circ}, 270^{\circ}$. In multiple scattering conditions (top part of Fig. 2) there clearly is a fair amount of energy at those angles. The subtraction of the higher order scattering energy found at those specific angles, when done at all azimuths, leads to a second order scattering pattern [4]. So, it appears that multiple scattering effects occurring in dense water cloud can be simplified to second 
order scattering. In the following, we will examine what are the mechanisms leading to depolarization of the lidar signal from second order scattering.

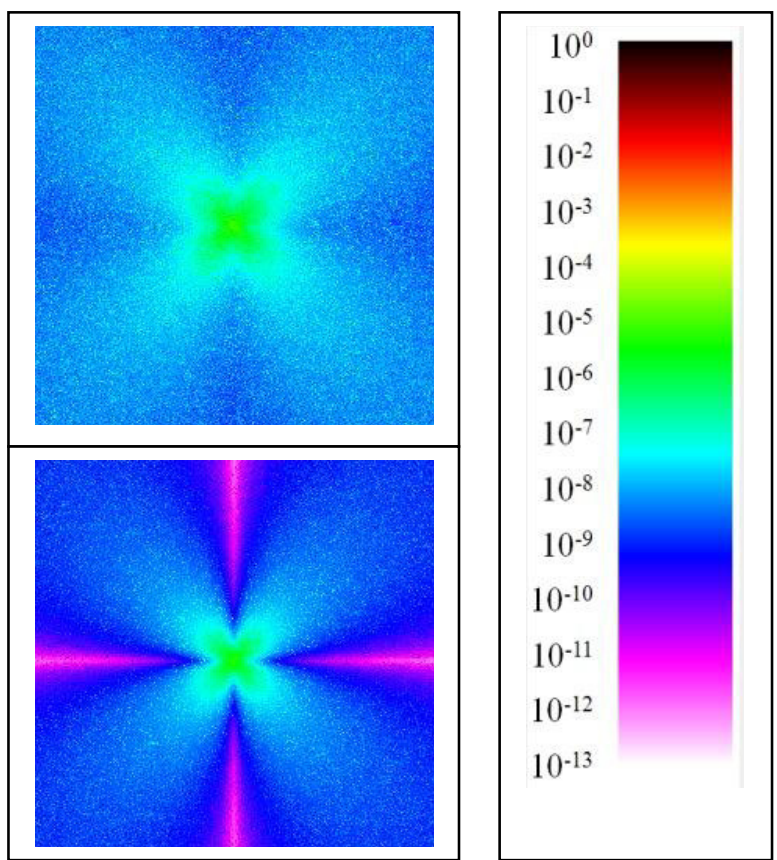

Figure 2. Monte Carlo simulation of cross polarization azimuthal pattern. The top image contains all orders scattering. The image at the bottom is for second order scattering only.

\section{BACKSCATTERING AND DEPOLARIZATION}

Lidars with polarization capabilities usually work at a specific FOV and detect the parallel and cross polarization components of the lidar return. The lidar signals will contain all scattering orders. In this paper, we want to reduce multiple scattering effects to second order scattering. To do so, it is necessary to have a look at the Mie scattering phase function and how the scattered light is depolarized.

The linear depolarization ratio $\left(\delta_{\text {lin }}\right)$ at a scattering angle $\beta$ is defined as the ratio of the power integrated over the azimuthal angles $\varphi$ in the plane of polarization orthogonal $\left(I_{\perp}\right)$ and parallel $\left(I_{/ /}\right)$ to the linearly polarized source:

$$
\delta_{l i n}(\beta)=\frac{\left.<I_{\perp}\right\rangle}{<I_{/ /}>}=\frac{\int_{0}^{2 \pi} I_{y}(\beta, \varphi) d \varphi}{\int_{0}^{2 \pi} I_{x}(\beta, \varphi) d \varphi} .
$$

Using Mie theory, it can be shown that:

$$
\delta_{\text {lin }}(\beta)=\frac{\left(P_{2} \cos ^{2} \beta-2 P_{3} \cos \beta+P_{1}\right)}{\left(3 P_{2} \cos ^{2} \beta+2 P_{3} \cos \beta+3 P_{1}\right)},
$$

where in $P_{1}, P_{2}$ and $P_{3}$ are the Mie scattering phase matrix elements defined by Deirmendjian.

Fig. 3 shows the phase function and the depolarization ratio at a wavelength of $0.532 \mu \mathrm{m}$ for linear incident waves on water-droplets of effective radius of $6 \mu \mathrm{m}$. The water droplets size distribution is a gamma distribution with $\alpha=7$ and $\beta=1.5$.

For depolarization, the major observations are:

1) The light is not depolarized in the forward scattering direction for angles smaller than $20^{\circ} ; 2$ ) the depolarization is equal to 0 at $180^{\circ}$; 3) the depolarization increases very sharply around $180^{\circ}$.

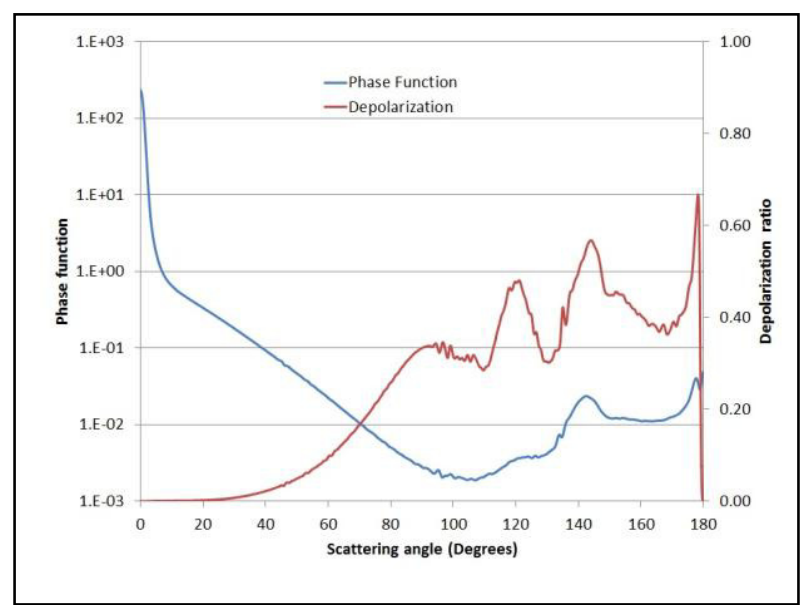

Figure 3. Phase functions and linear depolarization ratio for a water droplets gamma distribution with effective radius of $6 \mu \mathrm{m}$.

So, for a lidar, depolarization is mainly caused by a backscattering occurring after one or many forward scatterings as illustrated in Fig. 4.

A lidar with a given FOV $\left(\theta_{i}\right)$ will measure the backscattered light without any distinction regarding the forward scattering event position in space. The cross polarized second order scattering signal detected will be the sum of all those signals and it will be given by:

$$
P_{D \perp}\left(z_{c}, \theta_{i}\right)=P\left(z_{c}\right) \int_{0}^{z_{c} \beta_{i}} \int_{0} F(z, \beta) \sin \beta \mathrm{d} \beta \mathrm{d} z
$$




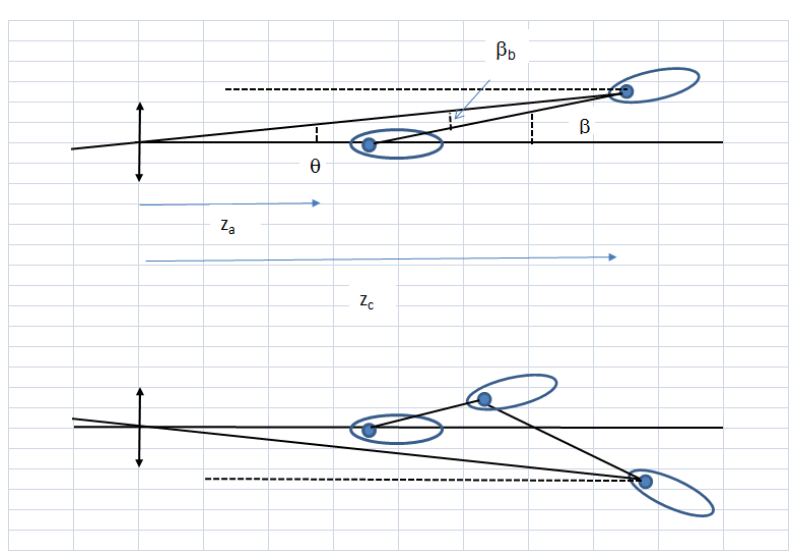

Figure 4. Double scattering processes and higher scattering processes leading to backscattering depolarization.

where $P\left(z_{c}\right)=P_{0} \exp \left[-2 \int_{0}^{z_{c}} \alpha(z) d z\right] \frac{c \tau}{2} \frac{4 \pi A}{z_{c}^{2}}$, and $F(z, \beta)=\left[\alpha_{s}(z) p(r, \beta)\right]\left[\alpha_{s}\left(z_{c}\right) p_{\perp}\left(r, \beta_{b}\right)\right]$,

where, $c, \tau$ and $A$ are respectively the speed of light, the pulse width, and the collecting optics area; $\alpha_{s}$ is the scattering extinction coefficient; $p(r, z, \beta)$ is the value of the phase function for the forward scattering $(\beta)$, and $p_{\perp}\left(r, z_{c}, \beta_{b}\right)$ the value of the perpendicular components of the phase function at backward scattering angles $\left(\beta_{b}=\pi-\beta+\theta\right)$ for a particle of radius $r ; z_{a}$ is where the cloud starts; $z_{c}$ is the distance where the scattered radiation is measured; the quantity $\left[\alpha_{s}(z) p(r, z, \beta)\right]$ represents the forward scattering coefficient while $\left[\alpha_{s}\left(z_{c}\right) p_{\perp}\left(r, z_{c}, \beta_{b}\right)\right]$ represents the cross polarization backscattering coefficient. The FOV $\theta$ is easily related to the scattering angle $\beta$ via: $\tan \beta=\left(z_{c} \tan \theta\right) /\left(z_{c}-z\right)$.

The forward scattering phase function is modelled as a summation of Gaussians [6]

$$
\begin{aligned}
& p(r, \beta)=\frac{1}{2} \frac{1}{\pi \beta_{d}} \exp \left(-\beta^{2} / \beta_{d}{ }^{2}\right)+\frac{A_{g}}{2} \frac{1}{\pi \beta_{g}} \exp \left(-\beta^{2} / \beta_{g}{ }^{2}\right) \\
& \beta_{d}=0.585\left(\lambda / 2 r_{e}\right) ; \beta_{g}=0.481 ; A_{g}=0.89 ;
\end{aligned}
$$

$r_{e}$ : effective radius, $\lambda$ : wavelength.

There is no existing model for $p_{\perp}\left(r, \beta_{b}\right)$, the perpendicular components of the phase function.
Our analysis has lead us rather to use the depolarization parameter $\left(D=p_{\perp} /\left(p_{\perp}+p_{/ /}\right)\right)$ rather than $p_{\perp}\left(r, \beta_{b}\right)$. Fig. 5 shows the definition of various parameters used to express the depolarization parameter as a function of scattering angles ranging from $170^{\circ}$ to $180^{\circ}$. The complete derivation of the parameterization for $r_{e}$ ranging from 2 to $12 \mu \mathrm{m}$ will be published in Appl. Opt in 2017. The mathematical representation is as follows:

$\beta_{\text {Max }}\left(r_{e}\right)=1.5845 \ln \left(r_{e}\right)+175.09^{\circ}$

If, $\beta \geq \beta_{\text {Max }}\left(r_{e}\right)$

$D\left(\beta, r_{e}\right)=A\left(r_{e}\right)\left[1-\exp -\left((\pi-\beta) /\left(\beta_{d}\left(r_{e}\right) w\left(r_{e}\right)\right)^{4}\right]\right.$

$w\left(r_{e}\right)=0.4625+0.10485 r_{e}$

If, $\beta<\beta_{\operatorname{Max}}\left(r_{e}\right)$

$D\left(\beta, r_{e}\right)=B_{1}\left(r_{e}\right) \cdot \exp \left(-B_{3}(0.944 \pi-(\pi-\beta))+B_{2}\left(r_{e}\right)\right.$ In short, $D\left(\beta, r_{e}\right)$ starts from a value of zero at $180^{\circ}$ and increases quickly following a superGaussian. It needs to be noted here that the width of the super-Gaussian is directly related to the width of the forward scattering peak $\beta_{d}$. Past the maximum value, in the direction of decreasing angles, $D\left(\beta, r_{e}\right)$ decreases exponentially.

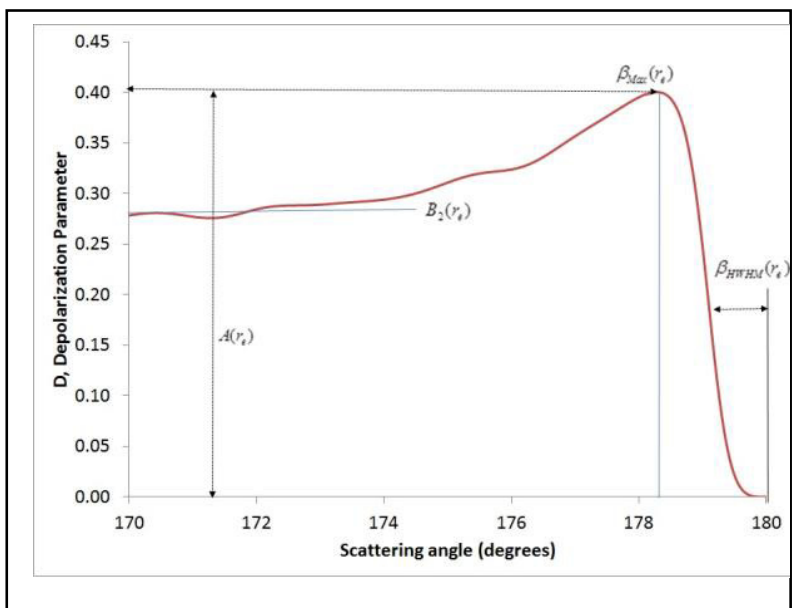

Figure 5. Parameterization of backscattering depolarization parameter. A water droplet cloud of effective radius of $6 \mu \mathrm{m}$ is represented.

\section{RESULTS}

Fig. 6 shows the calculated and normalized detected energy in rings delimited by FOV $\theta_{i}$ 
around the backscatter direction and for effective particle radius of $6 \mu \mathrm{m}$. It is obtained using the model previously described (see Fig. 5) for a wavelength of $532 \mathrm{~nm}$, beam divergence: $0.3 \mathrm{mrad}$, pulse width: $5 \mathrm{~ns}$, cloud base: $100 \mathrm{~m}$, depth: $20 \mathrm{~m}$, extinction coefficient equal to $0.25 \mathrm{~m}^{-1}$.

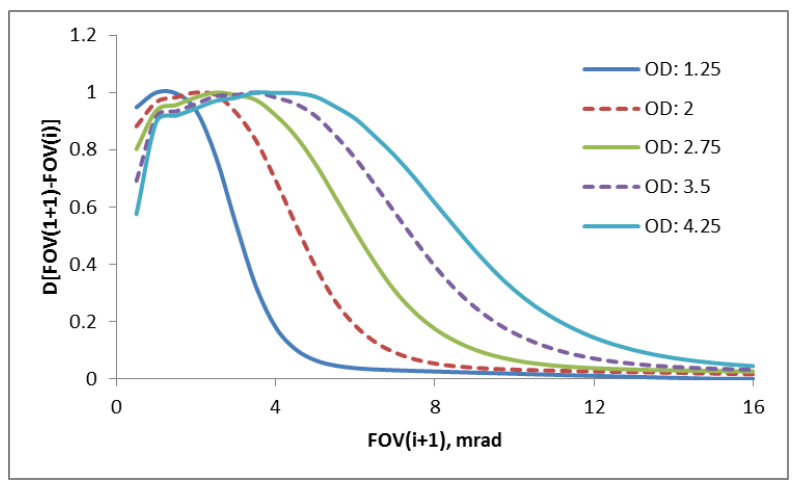

Figure 6. From $2^{\text {nd }}$ scattering polarimetric model. Detected energy in rings delimited by FOV $\theta_{i}$ and $\theta_{i+1}$ for effective particle radius $6 \mu \mathrm{m}$.

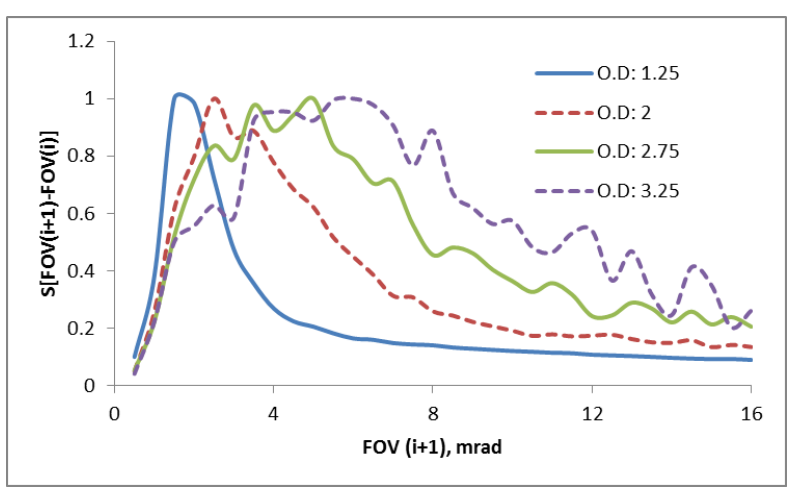

Figure 7. From MC simulation. Detected energy in rings delimited by FOV $\theta_{i}$ and $\theta_{i+1}$ for effective particle radius $6 \mu \mathrm{m}$

Using the same cloud parameters and lidar characteristics as for the model calculation displayed in Fig. 6, MC simulations were performed and images were analysed to recover second order scattering. Figure 7 shows the calculated and normalized detected energy in rings delimited by FOV $\theta_{i}$ and $\theta_{i+1}$. Fig. 8 shows a clear dependency of the polarimetric signal over the droplet size

The MC results are noisier for the larger optical depths. The MC results and polarimetry double scattering model agree reasonably well in shape.

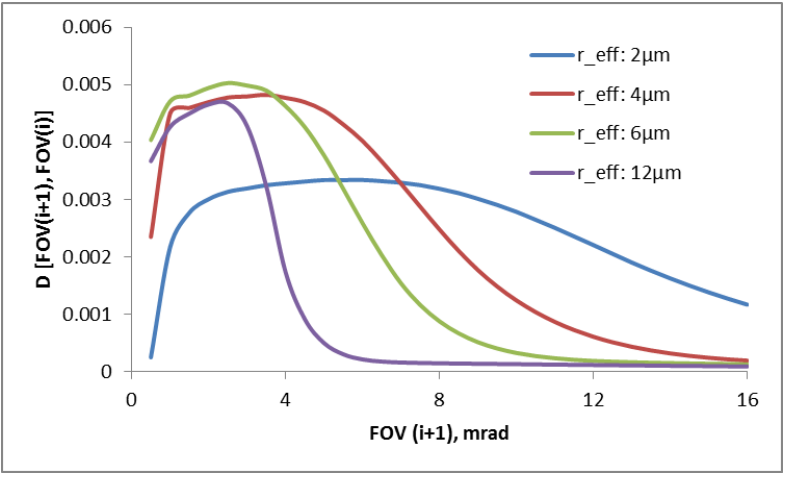

Figure 8 . From $2^{\text {nd }}$ scattering polarimetric model. Detected energy in ring delimited by FOV $\theta_{i}$ and $\theta_{i+1}$ for effective particle radius $2,4,6 \& 12 \mu \mathrm{m}$. Same cloud extinction and position as Fig. 6 and Fig. 7. Penetration depth is of $11 \mathrm{~m}$.

\section{CONCLUSION}

Monte Carlo simulations support experimental observations reported in [1]; i.e. multiple scattering cross polarization can be reduced to a second order scattering using the azimuthal dependency of the secondary polarization. A model of the polarimetric backscattering phase function has led to a mathematical expression for the polarimetric backscatter signal. This signal can be related to the droplet particle size. Future work will focus on the extraction of the droplet size.

\section{REFERENCES}

[1] S. R. Pal and A. I., Carswell, Polarization anisotropy in lidar multiple scattering from atmospheric clouds, Appl. Opt., 24, 3464-3471 (1985).

[2] V. Griaznov, I. Veselovskii, P. Girolamo, M. Korenskii and D. Summa, Spatial distribution of doubly scattered polarized laser radiation in the focal plane of a lidar receiver, Appl. Opt. 46, 6821-6830, (2007)

[3] N. Roy, G. Roy, L. R. Bissonnette and J-R. Simard, Measurement of the azimuthal dependence of cross-polarized lidar returns and its relation to optical depth, Appl. Opt. 43, 2777-2785, (2004)

[4] A. Doroshkevich, The effect of droplet cloudy microstructure on the polarization characteristics of double scattering lidar return, SPIE Vol. 10035, 1003550, (2016)

[5] G. Tremblay, G. Roy, High fidelity imaging algorithm for the Undique Monte Carlo simulator. 27rd International Laser Radar Conference, New York, USA, (2015)

[6] Luc R. Bissonnette, Gilles Roy, Laurent Poutier, Stewart G. Cober, and George A. Isaac, Multiple-scattering lidar retrieval method: tests on Monte Carlo simulations and comparisons with in situ measurements, Appl. Opt, 41, (2002) 